\title{
Meeting report: genome engineering, plant genetics and young scientist forum
}

\author{
Nam-Soo Kim • Seungman Kang
}

Received: 17 September 2014/ Accepted: 17 September 2014/Published online: 8 October 2014

(C) The Genetics Society of Korea and Springer-Science and Media 2014

\section{To the Editor,}

The 2014 Annual Meeting of the Genetics Society of Korea (GSK) was held at the Kunsan National University, Kunsan, Korea, in affiliation with the 69th Annual Meeting of the Korean Association of Biological Sciences (KABS) in August 12-13, 2014. Five societies are integrated in the KABS; the Korean Society of Integrative Biology (formerly the Zoological Society of Korea), the Ecological Society of Korea, the Korean Society of Biology Education, the Korean Society of Systematic Zoology, and the Genetics Society of Korea. At the 69th KABS meeting, over 400 posters were presented and each society organized several oral presentations. In the GSK meeting, three out of 86 posters were awarded the Springer Award. There were also two symposia (Symposium I-Genome Engineering; Symposium II-Crop Genetics) and one colloquium of young scientists held at the meeting.

In the Genome Engineering Symposium, three eminent genome editing scientists presented their recent progress in genome editing and engineering. The first speaker was Professor Jin-Soo Kim from Seoul National University, Korea. The title of his talk was "Genome editing in cultured cells and whole organisms with programmed nucleases". Genome editing allows targeted mutagenesis in higher eukaryotic cells and organisms. Thus, it is used in various areas of biology, biotechnology, and medicine. Prof. Kim's

\section{N.-S. Kim (ه)}

Department of Molecular Bioscience, Kangwon National

University, Korea, Chuncheon 200-701, Korea

e-mail: nsk6472@yahoo.ca; kimnamsu@kangwon.ac.kr

\section{S. Kang}

Division of Life Sciences, Korea University, Seoul 137-701, Korea group is a leader in genome editing. They developed zinc finger nucleases (ZFNs), transcription activator-like effector nucleases (TALENs), and Cas9 RNA-guided engineered nucleases (RGENs). The last technique, derived from the type II CRISP/Cas prokaryotic immune system, can cleave chromosomal DNA in a targeted manner by producing DNA double-strand breaks, which are repaired by endogenous systems, resulting in targeted genome modification. The RGEN system has several advantages over other systems that utilize DNA for delivery into cells for genome editing. The RGEN utilizes RNA and ribonucleoprotein (RNP) for delivery into cells, which may expedite the reaction between RGEN nuclease and the target sequence. This technique improves detection and reduces off-target binding by reducing the time that RGEN spends unbound in cells, resulting in reduced mosaicism when RGEN is applied to embryonic cells. Moreover, RGEN technique can be applied to detect mutations in vitro or in vivo in several cell types. Kim's group also analyzed mutational patterns around the RGEN target sites by non-homologous end joining (NHEJ) using mathematical algorithms. With this model, they can predict in-frame and out-of-frame knockout frequencies efficiently. Armed with experimental data of the off-target cleavage frequencies of various RGEN RNA structures, his team applied the RGEN technique to hemophilia. The RGEN technique promises to be an efficient tool for gene and cell therapy.

The second speaker was Professor Hyongbum Kim from Hanyang University, Seoul, Korea. His topic was "Efficient use of programmable nucleases for genome editing". Producing knock-out cells using engineered nucleases takes much effort and time. Prof. Kim developed a technique that causes RFP and GFP to be expressed only in mutated cells, which can reduce time and effort in the production of genome-edited cells. He obtained mutant cell 
populations that were enriched 10 - to 20 -fold mutant by selective sorting of the genome-edited cells using flow cytometry. However, this flow cytometric sorting system could potentially damage the cells. His team is currently developing a new reporter system that uses magnetic separation and antibiotic selection, which allows for the enrichment of genome-edited cells of various types with little to no damage. He proposed that this efficient biallelic mutation inducing system may open the possibility of gene therapy using engineered nucleases.

The last speaker was Dr. Eun-Ji Kim at Toolgen, Inc. in Seoul, Korea. Her topic was "Large-scale gene knock-out cell line development using RNA-Guided Engineered Nucleases (RGENs). The previous two talks had already introduced the innovative genome engineering technology utilized by programmable nucleases, which enable fast and efficient editing of genetic information in various cells and organisms. Moreover, the recently developed GREN technique, derived from a prokaryotic adaptive immune system CRISPR/CAS9, could provide a robust programmable nuclease platform with high reproducibility and specificity suitable for a large-scale production of geneedited cells and organisms. She presented a large scale gene knock-out cell line production pipeline by streamlining the knock-out and knock-in processes with a $90 \%$ success rate of RGEN. She demonstrated their highthroughput genome engineering process using HAP-1, a well-characterized human haploid cell line. They generated a collection of 311 isogenic knock-out cell lines, each with a specific mutation in non-essential kinase genes expressed in HAP-1 cells. She proposed that this high-throughput genome engineering approach may be utilized not only for production of knock-out cell lines, but also for drug screening and gene function analyses.

In the Crop Genetics Symposium, three plant geneticists presented their interesting results. The first speaker was Professor Nam-Chon Paek, Seoul National University, Korea. The title of his talk was "Mutation of NAC016 confers delayed senescence and abiotic stress tolerance in Arabidopsis". Ageing is an irrefutable process of life. Plant senescence is a highly ordered process and is the final stage of leaf development. In the process, senescence-associated genes (SAGs) execute an orderly process of dismantling the photosynthetic apparatus and remobilizing cellular components. Prof. Paek's group has worked a senNAC (senescence-associated Nam/ATAF1/2/CUC2) transcription factor, which promotes senescence in Arabidopsis. A senNac mutant, nac016, remained green under senescenceinducing conditions, and an NACO16 overexpressing (NAC016-OX) plant showed early senescence. In addition to its role in senescence, NACO16 also affects abiotic stress. Under salt and oxidative stress conditions, NACO16 expression rapidly increased in developing leaves, possibly to promote senescence. Under stress conditions, nac016 mutants stayed green, while the NACO16-OX plants senesced rapidly. Yeast one-hybrid assays showed that the NAC016 TF binds to the promoter of NAP and ORS1, from which they proposed that NACO16 regulatory mechanisms promoting leaf senescence exhibit crosstalk with salt and oxidative stress-responsive signaling pathways. Moreover, NAC016 was involved in drought stress responses; nac016 mutants revealed high drought tolerance whereas NAC016-OX plants showed low drought tolerance. Using genome-wide gene expression microarray analysis and MEME searches, they identified the NAC016specific binding motif (NAC16BM), GATTGGAT[AT]XA, in the promoters of genes up-regulated in the nac016-1 mutant and down-regulated in NAC016-OX plants. ABSCISIC ACID-RESPONSIVE ELEMENT BINDING PROTEIN1 (AREB1) encodes a central transcription factor in stress-responsive abscisic acid signaling pathway. The NAC016 directly binds to the NAC16BM in the $A R E B 1$ promoter and represses $A R E B 1$ transcription. NAC-LIKE, ACTIVATED BY AP3/PI (NAP) is a target gene of NAC016, and knockout of the NAP gene exhibited string drought tolerance. NAP binds to the AREBI promoter to suppress AREBI transcription. With these results, Prof. Paek proposed that a trifurcate feed-forward pathway involving NACO16, NAP, and AREBI functions in the drought stress response, in addition to affecting the leaf senescence in Arabidopsis.

The second speaker of Symposium II was Professor Jeong-Il Kim from Chunnam National University, Korea. His topic was "Functional analysis of novel Arabidopsis genes, AtKDAs that involves in blue light signaling". Sessile plants must adapt to the ambient environment. They recognize day and night and respond season changes. In order to respond these environmental changes, plants have acquired multiple highly complex and sophisticated photoreceptors to mediate light regulation of growth and development. Phytochromes are red/far red light receiving photoreceptors. Cryptochromes and phototropins are blue light receiving photoreceptors. Phytochromes and the genes regulated by phytochromes have been well-characterized by genetic and biochemical analyses, whereas studies on the blue-light receptors and their signaling are relatively meagre. In the analyses of phytochrome signaling in Arabidopsis, Prof Kim's group identified genes called AtKDAs that abolished completely the blue-light signaling when over-expressed. In previous research, Prof Kim's group developed several plants that suppressed a shade-avoidance phenotype by over-expression of phytochromes. When crops are grown in dense arrangements, the plants over-grow feebly in competition for light by shadeavoidance. But these shade avoidance-suppressed plants can avoid this over-growth phenotype. Kim's group 
isolated 87 genes that are highly expressed in these shade avoidance-suppressed plants compared to the control by microarray analysis. Surprisingly, overexpression of these genes abolished the blue-light signal transduction pathway completely. Furthermore, the molecular functions of these genes were linked with cryptochromes. Because plants that over-expressed these genes exhibited increased plant height and size of leaves, stems, and flowers, they named these genes "kDa," which, pronounced in Korean means "enlargement". AtKDAs are present in two copies in various plants, including Arabidopsis, and are plant-specific, because no homologous genes were found in other kingdoms. AtKDAs revealed light-responsive gene expression patterns; they were induced by light, but suppressed by darkness. AtKDA1 was present in both the nucleus and the cytoplasm of cells, whereas AtKDA2 was present only in the nucleus. When the expression of AtKDAs was attenuated by RNAi, the blue-light signal pathways were increased. Yeast-two hybrid analysis revealed that the At$K D A$ genes could bind with the blue-light receptor cryptochrome. Expression of AtKDAs was induced under the blue light and subsequently interacted with cryptochromes. Thus, Prof Kim proposed that AtKDAs can function as negative regulators of the blue-light signaling pathways of cryptochromes.

The last speaker of Symposium II was Professor KiHong Jung from Kyunghee University, Korea with the topic of "Current stage of resources for phenomics in rice and a case study for photosynthesis". Phenomics is a recent addition to "omics". Plant phenotyping is the comprehensive assessment of complex plant traits and phenomics is a biology field that measures the total phenomes in response to a genetic mutation of environmental influence by high-throughput measurement systems. Although development in phenomics is relatively slow, Prof. Jung summarized the initiative on phenomics in plant biology in Korea. He argued that Korea needs strategic differentiation of phenomics research in order to catch up forerunning groups. Rice has served as a model organism in phenomics research. Several Korean groups have generated hundreds of thousands of T-DNA or Ds transposon insertion mutants in rice, resulting in the biggest mutant collections in the world. Moreover, a microarray database derived from transcriptomes of about 5,000 mutants can be directly applied to link the mutants to phenomic analyses, which covers about $60 \%$ of rice genes. Thus, the massive mutant library and the categorization based on trait characteristics can be harnessed to maximize phenomic analysis in rice. Prof. Jung's group has applied meta-expression profiles in conjunction with the microarray profiles to characterize gene expression in the mutants at different developmental stages, as well as in biotic and abiotic environmental challenges. From these analyses, they isolated genes expressed in roots, genes involved in photosynthesis, and stress response genes such as those for drought, high-salt, and phosphorous deficient conditions toward the ultimate goal of developing high-quality rice cultivars through phenomics approaches. From their previous research, they isolated many genes that regulate photosynthesis by microarray analysis and obtained 37 T-DNA insertion mutants in those photosynthesis-regulating genes. Of the 37 mutants, 10 mutant lines revealed a reduction in chlorophyll biosynthesis and subsequent growth retardation resulting from the low rate of photosynthesis. These results demonstrate that the target-oriented mutant analyses based on bioinformatics information were quite efficient at isolating genes for agronomic purposes. He surmised that the phenomics approach on the traits of assorted mutant lines via reverse genetics may maximize the efficiency of isolating genes of interest.

The participation of young scientists at this meeting is pivotal for the sustainable input and rejuvenation of the Genetics Society of Korea (GSK). Thus, GSK opened a forum of young scientists, in which 3 graduate students and a post-doc present their research progress to the audience. The first speaker was Dr. Hyunjoo Kim, a post-doctoral fellow at Seoul National University in Seoul, Korea. She presented a talk entitled "The DUSP26 phosphatase activator adenylate kinase 2 regulates FADD phosphorylation and cell growth". Adenylate kinase 2 (AK2), which balances the adenine nucleotide pool, is a multi-functional protein. She provided concrete evidence that AK2 negatively regulates tumor cell growth. AK2 formed a complex with DUSP26 phosphatase and stimulated DUSP26 activity independently of its adenylate kinase activity. AK2/ DUSP26 phosphatase protein complex dephosphorylated

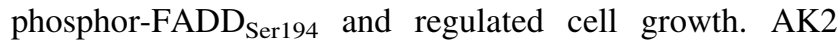
deficiency enhanced cell proliferation and induced tumor formation in a xenograft assay. This anti-growth function of AK2 was associated with its DUS26-stimulating activity. Down-regulation of AK2 is frequently found in tumor cells and human cancer tissues, showing high level of

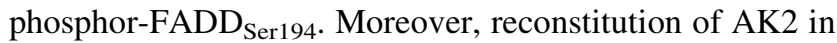
AK2-deficient tumor cells slowed cell proliferation and tumorigenesis. $\mathrm{AK}^{+/-}$mouse embryo fibroblasts exhibited enhanced cell proliferation with significant alteration in phosphor-FADD ${ }_{\text {Ser191. With these results, she suggested }}$ that AK2 is an associated activator of DUSP26 and suppresses cell proliferation via FADD dephosphorylation, postulating AK2 as a negative regulator of tumor growth.

The second speaker, Miss Se-Na Lee, a graduate student with Professor Sun-He Leem at Dong-A University in Busan, Korea, gave a talk entitled "Elevated levels of $E 1 F 1, E Z H 2$, and $S U Z 12$ lead to invasive progression of superficial bladder cancers". The rate of bladder cancer occurrence is increasing in aging societies. Although 
superficial bladder cancer is non-invasive and associated with a good prognosis after receiving treatment, about 20-50 \% of patients progress to invasive tumors, with a poor survival rate within 5 years. Previously, Prof. Leem's group had reported that E2F1 and its downstream effectors $E Z H 2$ and $S U Z 12$ could be important mediators for the invasive and metastatic progression of superficial tumors. Thus, Miss Lee presented in vivo and in vitro evidence that show how these genes affect the invasive and metastatic progression of superficial tumors. She used four cell lines total: two cells lines were superficial and two were invasive. In qRT-PCR and western blot analyses, both mRNA and protein expression of E2F1, EZH2, and SUZ12 were significantly lower in superficial bladder cancer than those in invasive bladder cancer cell lines. Cells overexpressing these genes showed increased invasiveness and migration ability, whereas the knock-down of these genes revealed a significant decrease in invasiveness and migratory ability of superficial bladder cells. Moreover, knock-down of E2F1, EZH2, and SUZ12 in invasive cancer cells decreased tumor size in a xenograft assay. By promoter -binding and ChIP assays, Miss Lee demonstrated that E2F1 controls the expression of EZH2 and SUZ12 through direct binding on their promoter regions. Miss Lee proposed that the expression levels of $E 2 F 1, E Z H 2$ and $S U Z 12$ can be used as predictive markers for poor prognosis in bladder tumors.

The third speaker was Mr. Jeong-An Gim, a graduate student with Heui-Soo Kim at Pusan National University in Busan, Korea. The title of his talk was "Genome-wide DNA methylation analysis before and after exercise in thoroughbred horses via MeDIP-Seq". Athletic performance may be the most important or ultimate criterion in selection of superior horses in race horse breeding. $\mathrm{Mr}$. Gim presented interesting experimental results on the exercise-related epigenetic processes in race horses. Among the epigenetic mechanisms, DNA methylation is a key mechanism regulating gene expression in response to environmental changes. He carried out a comparative genomic analysis of genome-wide DNA methylation profiles in the blood samples of two different thoroughbred horses before and after exercise. He chose two thoroughbred horses that were different in racing records and took a blood sample from each animal prior to exercise and after 30 min running. He checked methylation profiles of the DNA from the four samples by methylated-DNA immunoprecipitation sequencing (MeDIP-Seq). Interestingly, many genes revealed methylation differences between racing superior and inferior horses after running. After exercise, 596 genes were hypomethylated and 715 genes were hypermethylated in the racing superior horse, whereas 868 genes were hypomethylated and 794 genes were hypermethylated in racing inferior horse. He then analyzed these differentially methylated genes based on gene ontology (GO) annotations and compared the exerciserelated pathway patterns between the two horses. Genes related to cell division and adhesion were hypermethylated in the superior horse, whereas genes involved in cell signaling and transport were hypermethylated in the inferior horse. Analysis of the distribution of methylated $\mathrm{CpG}$ islands confirmed that hypomethylation occurred in genebodies after exercise. Long interspersed nuclear elements (LINEs) have abundant DNA methylation regions (DMRs). The DMRs in LINEs showed methylation changes after exercise. With these results, Mr. Gim posited that his results may serve as a basis in studying exercised-based reprogramming of epigenetic traits.

The last speaker in the forum was Mr. Hui-Gwan Goo, a graduate student of Professor Seungman Kang at Korea University in Seoul, Korea. Mr. Goo gave a talk entitled: "HtrA2/Omi deficiency causes instability of mtDNA and mitochondrial homeostasis proteins". High-temperature requirement protein $\mathrm{A} 2$ (HtrA2) is a serine protease and localizes in the mitochondria. The HtrA2 (also known as Omi) has diverse cellular roles, including mitochondrial homeostasis maintenance and regulation of cellular apoptosis. HtrA2 was known to be associated with many neurodegenerative diseases. However, the function of HtrA2 in mitochondria under normal condition has not been elucidated. Mr. Goo presented experimental results suggesting that mitochondrial DNA conformational stability is related with HtrA2. In motor neuron degeneration 2 (nmd2) mice, which have greatly reduced HtrA2 protease activity caused by a Ser276Cys missense mutation, conformational changes were apparent in the mitochondrial DNA from brain tissue of the $n m d 2$ mice. Overexpression of the HtrA2 showed restoration of the mtDNA conformational stability in $\mathrm{HtrA} 2^{-1-}$ MEF cells. Using $\mathrm{H}_{2} \mathrm{O}_{2}$ and NAC, Mr. Goo showed that the HtrA2 protease maintained mtDNA conformational stability by controlling ROS generation. He also showed that nuclear-encoded mtDNA repair genes, including POLG2, Twinkle, and ATPX1, were significantly up-regulated in the $\mathrm{HtrA} 2^{-l-}$ cells. Additionally, the mitochondrial proteins LON protease 1 (LONP1) and prohibitin (PHB) were over-expressed in the $\mathrm{HtrA} 2^{-1-}$ mouse embryonic fibroblast cells and in HtrA2 knockdown HEK2933T cells. The over-expressed LONP1 and PHB were regulated by active HtrA 2 proteases localized in the mitochondria of $\mathrm{HtrA} 2^{-1-}$ cells. Using immunoprecipitation, in vitro cleavage and co-transfection, he demonstrated that PHB interacts with and is directly cleaved by HtrA2. PHB is a mitochondrial inner membrane-associated protein and LONP1 is localized in the mitochondrial matrix. Thus, his hypothesis is that the mitochondrial inner membrane protein HtrA2 might directly interact with PHB, but not with LONP1. The intracellular ATP level is 
decreased in $\mathrm{HtrA} 2^{-/-}$cells compared to $\mathrm{HtrA}_{2}^{+/+}$cells, suggesting that HtrA2 deficiency causes a decrease in the mitochondrial membrane potential. Furthermore, reactive oxygen species (ROS) generation is greater in $\mathrm{HtrA}^{-1-}$ cells than in $\mathrm{HtrA} 2^{+/+}$cells. Thus, he concluded that $\mathrm{HtrA} 2$ is crucial in regulating mitochondrial homeostasis. 